\title{
A SHORT PROOF OF HÖLDER CONTINUITY FOR FUNCTIONS IN DEGIORGI CLASSES
}

\author{
Colin Klaus* and Naian Liao ${ }^{\dagger}$ \\ Ohio State University, The Mathematical Biosciences Institute \\ Columbus, OH 43210, U.S.A.; klaus.68@mbi.osu.edu \\ Chongqing University, College of Mathematics and Statistics \\ Chongqing, 401331, P. R. China; liaon@cqu.edu.cn
}

\begin{abstract}
The goal of this note is to give an alternative proof of local Hölder continuity for functions in DeGiorgi classes based on an idea of Moser.
\end{abstract}

\section{Introduction and main results}

Let $E$ be an open set in $\mathbf{R}^{N}$ and $K_{\rho}(y)$ be a cube of edge $2 \rho$ centered at $y \in \mathbf{R}^{N}$. When $y=0$ we simply write $K_{\rho}$. The DeGiorgi classes $[D G]_{p}^{ \pm}(E ; \gamma)$, with some $p>1$ and $\gamma>0$, consist of functions $u \in W_{\text {loc }}^{1, p}(E)$ satisfying

$$
\int_{K_{\rho}(y)}\left|D(u-k)_{ \pm}\right|^{p} d x \leq \frac{\gamma}{(R-\rho)^{p}} \int_{K_{R}(y)}\left|(u-k)_{ \pm}\right|^{p} d x
$$

for any pair of cubes $K_{\rho}(y) \subset K_{R}(y) \subset E$, and all $k \in \mathbf{R}$. We further define

$$
[D G]_{p}(E ; \gamma)=[D G]_{p}^{+}(E ; \gamma) \cap[D G]_{p}^{-}(E ; \gamma)
$$

In the sequel, we refer to the set of parameters $\{p, \gamma, N\}$ as the data and use $C$ as a generic constant that can be quantitatively determined apriori only in terms of the data.

For a function $u \in[D G]_{p}(E ; \gamma)$ and $K_{2 \rho}(y) \subset E$, we set

$$
\mu^{+}=\underset{K_{2 \rho}(y)}{\operatorname{ess} \sup } u, \quad \mu^{-}=\underset{K_{2 \rho}(y)}{\operatorname{essinf}} u, \quad \omega(2 \rho)=\underset{K_{2 \rho}(y)}{\operatorname{ess} \operatorname{osc}} u=\mu^{+}-\mu^{-} .
$$

When there is no ambiguity, we scratch "ess" in the following.

Now we state the following celebrated theorem of DeGiorgi, c.f. $[1,3,7]$.

Theorem 1.1. (I) If $u \in[D G]_{p}^{ \pm}(E ; \gamma)$, then there is a constant $C>0$ depending only on the data, such that

$$
\sup _{K_{\rho}(y)}(u-k)_{ \pm} \leq \frac{C}{(R-\rho)^{N}} \int_{K_{R}(y)}(u-k)_{ \pm} d x
$$

for any pair of cubes $K_{\rho}(y) \subset K_{R}(y) \subset E$ and all $k \in \mathbf{R}$.

https://doi.org/10.5186/aasfm.2018.4354

2010 Mathematics Subject Classification: Primary 35B65; Secondary 35J62, 35J92, 49N60.

Key words: DeGiorgi classes, Hölder continuity, Moser's method.

* Author is grateful to the Mathematical Biosciences Institute for partially supporting this research. MBI receives funding through the National Science Foundation grant DMS 1440386.

${ }^{\dagger}$ Corresponding author. Partially supported by the National Natural Science Foundation of China grant No. 11701054. 
(II) If $u \in[D G]_{p}(E ; \gamma)$, there are constants $C>0$ and $0<\alpha<1$ depending only on the data, such that for every pair of cubes $K_{\rho}(y) \subset K_{R}(y) \subset E$, we have

$$
\omega(\rho) \leq C \omega(R)\left(\frac{\rho}{R}\right)^{\alpha}
$$

Although the DeGiorgi classes were originally modelled after linear elliptic equations with bounded and measurable coefficients, DeGiorgi's approach to prove local boundedness and local Hölder continuity of their solutions made no reference to any equation, and such functional classes are general enough to include local minima or Q-minima of rather general functionals, which may not admit an Euler equation. A similar theorem, regarding the local boundedness and local Hölder continuity of solutions to quasilinear elliptic equations, was proved by Moser in [8]; see also [9], [7, Chap. 9]. However, the original proof of Moser kept referring to the equation.

It has been noted in [5] that Moser's idea can be employed to show local boundedness of functions in $[D G]_{p}^{+}(E ; \gamma)$, i.e., Part (I) of Theorem 1.1. Thanks to the recent remarks in [4] on properties of DeGiorgi classes, we are able to give an alternative proof of local Hölder continuity for functions in $[D G]_{p}(E ; \gamma)$ based on Moser's idea [8]. This is the main goal of this note. For a somewhat analogous approach see also [6].

Acknowledgement. The authors thank Professor Emmanuele DiBenedetto for suggesting the argument in this note and Professor Ugo Gianazza for discussions and remarks, which greatly helped to improve the final version of the note.

\section{Some lemmas}

The generalized DeGiorgi Classes $[G D G]_{p}^{ \pm}(E ; \gamma)$ are the collection of functions $u \in W_{\text {loc }}^{1, p}(E)$, for some $p>1$, satisfying

$$
\int_{K_{\rho}(y)}\left|D(u-k)_{ \pm}\right|^{p} d x \leq \frac{\gamma}{(R-\rho)^{p}}\left(\frac{R}{R-\rho}\right)^{N p} \int_{K_{R}(y)}\left|(u-k)_{ \pm}\right|^{p} d x .
$$

It is noteworthy that the conclusion of Theorem 1.1, Part (I) still holds for functions in $[G D G]_{p}^{+}(E ; \gamma)$. One just has to note that the extra term on the right-hand side of the definition of $[G D G]_{p}^{+}(E ; \gamma)$ is "homogeneous" with respect to the diameter of the cubes, then one could repeat the proof in [3, Theorem 2.1, Chap. 10], [5, Lemma 2.1] or [7, Lemma 5.4, Chap. 2].

For ease of notation, we write $\omega=\omega(2 \rho)$. We introduce two functions that are due to Moser [8]:

$$
w_{1}=\varphi_{1}(u)=\ln \frac{\omega}{2\left(\mu^{+}-u\right)}, \quad w_{2}=\varphi_{2}(u)=\ln \frac{\omega}{2\left(u-\mu^{-}\right)} .
$$

Lemma 2.1. Suppose $u \in[D G](E ; \gamma)$. The two functions $w_{1+}$ and $w_{2+}$ are both in $[G D G]_{p}^{+}(E ; \bar{\gamma})$ for some $\bar{\gamma}$ depending only on the data.

Proof. Since $\varphi_{1+}:\left(-\infty, \mu^{+}\right) \rightarrow \mathbf{R}$ is convex and non-decreasing, the function $w_{1+} \in[G D G]_{p}^{+}(E ; \bar{\gamma})$ by Lemma 2.1 in [4]. Since $\varphi_{2+}:\left(\mu^{-}, \infty\right) \rightarrow \mathbf{R}$ is convex, non-increasing and vanishes for $u \geq \mu^{-}+\frac{\omega}{2}$, the function $w_{2+} \in[G D G]_{p}^{+}(E ; \bar{\gamma})$ by Lemma 2.2 in [4].

Next we need a lemma whose proof can be found on p. 356 of [3] or p. 54 of [7]. 
Lemma 2.2. Let $v \in W^{1,1}\left(K_{r}(y)\right)$ and assume that the set $[v=0]$ has positive measure. There exists a positive constant $C$ depending only on $N$, such that

$$
\int_{K_{r}(y)}|v| d x \leq C \frac{r^{N+1}}{|[v=0]|} \int_{K_{r}(y)}|D v| d x .
$$

Finally, we recall Proposition 3.3 from [4].

Lemma 2.3. Let $u \in[D G]_{p}^{-}(E ; \gamma)$ be non-negative and bounded above by a positive constant $M$. Then

$$
\int_{K_{\rho}(y)}|D \ln u|^{p} d x \leq \frac{\gamma p}{(R-\rho)^{p}} \int_{K_{R}(y)} \ln \frac{M}{u} d x
$$

for any pair of cubes $K_{\rho}(y) \subset K_{R}(y) \subset E$.

\section{Proof of Hölder continuity}

Without loss of generality, we may take $y=0$. Let us first apply Lemma 2.3 to $w_{1}$ and $w_{2}$. Indeed, since $u \in[D G]_{p}(E ; \gamma)$ we have both $\mu^{+}-u$ and $u-\mu^{-}$members of $[D G]_{p}^{-}(E ; \gamma)$. Therefore, Lemma 2.3 yields

$$
\int_{K_{\rho}}\left|D \ln \frac{\omega}{2\left(\mu^{+}-u\right)}\right|^{p} d x \leq \frac{\gamma}{(R-\rho)^{p}} \int_{K_{R}} \ln \frac{\omega}{\mu^{+}-u} d x
$$

that is,

$$
\int_{K_{\rho}}\left|D w_{1}\right|^{p} d x \leq \frac{\gamma}{(R-\rho)^{p}} \int_{K_{R}}\left|w_{1}\right| d x+\gamma \frac{R^{N}}{(R-\rho)^{p}} .
$$

Similarly, we have

$$
\int_{K_{\rho}}\left|D w_{2}\right|^{p} d x \leq \frac{\gamma}{(R-\rho)^{p}} \int_{K_{R}}\left|w_{2}\right| d x+\gamma \frac{R^{N}}{(R-\rho)^{p}} .
$$

Now we go with two alternatives: either

$$
\left|\left[u \leq \mu^{+}-\frac{\omega}{2}\right] \cap K_{\rho}\right| \geq \frac{1}{2}\left|K_{\rho}\right| \quad \text { or } \quad\left|\left[u \geq \mu^{-}+\frac{\omega}{2}\right] \cap K_{\rho}\right| \geq \frac{1}{2}\left|K_{\rho}\right| .
$$

In terms of $w_{1}$ and $w_{2}$, this may be rephrased as either

$$
\left|\left[w_{1} \leq 0\right] \cap K_{\rho}\right| \geq \frac{1}{2}\left|K_{\rho}\right| \quad \text { or } \quad\left|\left[w_{2} \leq 0\right] \cap K_{\rho}\right| \geq \frac{1}{2}\left|K_{\rho}\right| .
$$

Suppose the first alternative is in force, the second alternative being similar. We may employ Lemma 2.2 and the fact that $w_{1} \geq-\ln 2$ to obtain that

$$
\int_{K_{\rho}}\left|w_{1}\right| d x=\int_{K_{\rho}} w_{1+} d x+\int_{K_{\rho}} w_{1-} d x \leq C \rho \int_{K_{\rho}}\left|D w_{1+}\right| d x+C \rho^{N} .
$$

The integral term on the right-hand side is estimated by Hölder's inequality, Young's inequality and (3.1) as

$$
\begin{aligned}
C \rho \int_{K_{\rho}}\left|D w_{1+}\right| d x & \leq C \rho^{1+N-\frac{N}{p}}\left(\int_{K_{\rho}}\left|D w_{1+}\right|^{p} d x\right)^{\frac{1}{p}} \\
& \leq C \rho^{1+N-\frac{N}{p}}\left(\frac{\gamma}{(R-\rho)^{p}} \int_{K_{R}}\left|w_{1}\right| d x+\gamma \frac{R^{N}}{(R-\rho)^{p}}\right)^{\frac{1}{p}}
\end{aligned}
$$




$$
\leq C \frac{\rho^{1+N-\frac{N}{p}}}{R-\rho}\left(\int_{K_{R}}\left|w_{1}\right| d x\right)^{\frac{1}{p}}+C \frac{\rho^{1+N}}{R-\rho}\left(\frac{R}{\rho}\right)^{\frac{N}{p}} .
$$

Thus we obtain

$$
\int_{K_{\rho}}\left|w_{1}\right| d x \leq C \frac{\rho^{1+N-\frac{N}{p}}}{R-\rho}\left(\int_{K_{R}}\left|w_{1}\right| d x\right)^{\frac{1}{p}}+C \frac{\rho^{1+N}}{R-\rho}\left(\frac{R}{\rho}\right)^{\frac{N}{p}}+C \rho^{N} .
$$

An interpolation argument (see [2, Lemma 4.3, Chap. I]) yields that

$$
\frac{1}{\rho^{N}} \int_{K_{\rho}}\left|w_{1}\right| d x \leq C(\text { data }) \text {. }
$$

An application of Lemma 2.1 gives that $w_{1+} \in[G D G]_{p}^{+}(E ; \bar{\gamma})$. As a result, Theorem 1.1, Part (I) holds for $w_{1+}$. The supreme estimate together with (3.3) yields that

which implies

$$
\sup _{K_{\frac{\rho}{2}}} w_{1+} \leq C f_{K_{\rho}} w_{1+} d x \leq C(\text { data })
$$

Therefore

$$
\underset{K_{\frac{\rho}{2}}}{\operatorname{ess} \sup } u \leq \mu^{+}-\frac{1}{2 e^{C}} \omega \text {. }
$$

$$
\underset{K_{\frac{\rho}{2}}}{\operatorname{ess} \text { osc }} u \leq\left(1-\frac{1}{2 e^{C}}\right) \omega .
$$

A standard iteration finishes the proof.

\section{References}

[1] De Giongi, E.: Sulla differenziabilità e l'analiticità degli integrali multipli regolari. - Mem. Accad. Sci. Torino Cl. Sci. Fis. Mat. Natur. 3:3, 1957, 25-43.

[2] DiBenedetto, E.: Degenerate parabolic equations. - Universitext, Springer-Verlag, New York, 1993.

[3] DiBenedetto, E.: Partial differential equations. Second edition. Cornerstones. - Birkhäuser Boston, Inc., Boston, MA, 2010.

[4] DiBenedetto, E., and U. Gianazza: Some properties of De Giorgi classes. - Rend. Istit. Mat. Univ. Trieste 48, 2016, 245-260.

[5] DiBenedetto, E., and N.S. Trudinger: Harnack inequalities for quasi-minima of variational integrals. - Ann. Inst. H. Poincaré Anal. Non Linéaire 1:4, 1984, 295-308.

[6] DÜzGün, F. G., P. Marcellini, and V. Vespri: An alternative approach to the Hölder continuity of solutions to some elliptic equations. - Nonlinear Anal. 94, 2014, 133-141.

[7] Ladyzhenskaya, O. A., and N. N. Ural'tseva: Linear and quasilinear elliptic equations. Academic Press, New York, 1968.

[8] Moser, J.: A new proof of de Giorgi's theorem concerning the regularity problem for elliptic differential equations. - Comm. Pure Appl. Math. 13, 1960, 457-468.

[9] NAsh, J.: Continuity of solutions of parabolic and elliptic equations. - Amer. J. Math. 80, $1958,931-954$. 